\title{
Novel polygodial analogs P3 and P27: Efficacious therapeutic agents disrupting mitochondrial function in oral squamous cell carcinoma
}

\author{
JORGE DE LA CHAPA ${ }^{1}$, PRAJJAL KANTI SINGHA ${ }^{2}$, McKAY SALLAWAY $^{1}$, KRISTEN SELF $^{1}$, \\ RANNA NASRELDIN $^{1}$, RAMESH DASARI ${ }^{3}$, MATTHEW HART ${ }^{4,5}$, ALEXANDER KORNIENKO $^{3}$, \\ JEREMY JUST $^{6}$, JASON A. SMITH ${ }^{6}$, ALEX C. BISSEMBER ${ }^{6}$ and CARA B. GONZALES ${ }^{1,5}$
}

\begin{abstract}
${ }^{1}$ Department of Comprehensive Dentistry, University of Texas Health Science Center at San Antonio School of Dentistry;
${ }^{2}$ Department of Pathology, University of Texas Health Science Center at San Antonio School of Medicine, San Antonio, TX 78229-3900; ${ }^{3}$ Department of Chemistry and Biochemistry, Texas State University, San Marcos, TX 78666-4684;

${ }^{4}$ Center for Innovation in Drug Discovery High Throughput Facility; ${ }^{5}$ Cancer Therapy and Research Center,

University of Texas Health Science Center at San Antonio, San Antonio, TX 78229-3900, USA;

${ }^{6}$ School of Physical Sciences - Chemistry, University of Tasmania, Hobart, Tasmania 7001, Australia
\end{abstract}

Received November 22, 2017; Accepted July 3, 2018

DOI: $10.3892 /$ ijo.2018.4585

\begin{abstract}
Polygodial, a drimane sesquiterpenoid dialdehyde isolated as a pungent component of the water pepper Persicaria hydropiper, exhibits antifeedant, antimicrobial, anti-inflammatory and anticancer effects. Polygodial also activates transient receptor potential vanilloid subtype 1 (TRPV1) channels. Previously, we described the synthesis of a $\mathrm{C}_{12}$-Wittig derivative of polygodial, termed $\mathrm{P} 3$, with significant antiproliferative effects against multiple cancer types including oral squamous cell carcinoma (OSCC). In the present study, a more potent derivative, P27, with superior anti-proliferative effects in vitro and antitumor effects in Cal-27 derived xenografts is described. Polygodial, P3, and P27 all significantly decreased OSCC tumor growth, with P27 being equipotent with polygodial and P3 being the least efficacious. However, neither analog elicited the adverse effect observed with polygodial: Profound transient inflammation. Although P3 and P27 pharmacophores are based on polygodial, novel effects on OSCC cell cycle distribution were identified and shared anticancer effects that are independent of TRPV1 activity were observed.
\end{abstract}

Correspondence to: Dr Cara B. Gonzales, Department of Comprehensive Dentistry, University of Texas Health Science Center at San Antonio School of Dentistry, 7703 Floyd Curl Drive, MCS 8258, San Antonio, TX 78229-3900, USA

E-mail: gonzalesc5@uthscsa.edu

Abbreviations: OSCC, oral squamous cell carcinoma; TRPV1, transient receptor potential channel vanilloid subtype 1

Key words: polygodial, oral squamous cell carcinoma, mitochondrial dysfunction, apoptosis
Polygodial elicits an S-phase block, whereas P3 and P27 lead to $\mathrm{G}_{2} / \mathrm{M}$ phase arrest. Pretreatment of OSCC cells with the TRPV1 antagonist capsazepine does not affect the antiproliferative activity of $\mathrm{P} 3$ or $\mathrm{P} 27$, indicating that TRPV1 interactions do not regulate OSCC cell proliferation. Indeed, calcium imaging studies identified that the analogs neither activate nor antagonize TRPV1. Behavioral studies using a rat model for orofacial pain confirmed that these analogs fail to induce nocifensive responses, indicating that they are non-noxious in vivo. All compounds induced a significant concentration-dependent decrease in the mitochondrial transmembrane potential and corresponding apoptosis. Considering that P27 is equipotent to polygodial with no TRPV1-associated adverse effects, P27 may serve as an efficacious novel therapy for OSCC.

\section{Introduction}

Head and neck squamous cell carcinoma (HNSCC) is the sixth most common cancer worldwide and the eighth most common cancer in the USA, with an incidence that is everincreasing (1). The majority of these cancer cases $(60 \%)$ are identified within the oral cavity (oral squamous cell carcinoma or OSCC), usually in the base of the tongue or the floor of the mouth (1). In total $\sim 70 \%$ of newly diagnosed OSCC cases are late-stage disease (stage III and IV) with a 5-year overall survival rate reportedly as low as $34 \%$ (1). Therefore, novel therapeutic strategies to target advanced OSCC are required to treat this deadly disease. Thus, the aim of the present study was to synthesize novel polygodial analogs and investigate their cytotoxic effects against multiple drug-resistant cancer cell lines in vitro (2). Polygodial, a known transient receptor potential vanilloid subtype 1 (TRPV1) agonist, is an unsaturated 1,4-dialdehyde sesquiterpene and the pungent component of peppers such as the Dorrigo pepper, mountain pepper and water pepper (3-6). Polygodial has a wide range of 
pharmacological activities including anti-inflammatory, antimicrobial and anticancer effects (6-9). Previously described was the synthesis of a $\mathrm{C}_{12}$-Wittig derivative of polygodial, herein termed $\mathrm{P} 3$, which demonstrated antiproliferative potencies that were 4-fold higher than those of polygodial against a panel of cancer cell lines (A549, SKMEL-28, MCF-7, U373 and Hs683) (2). Notably, these antiproliferative effects were via a TRPV1-independent mechanism of action that has yet to be identified. In the present study, an additional analog, P27, was synthesized that has more marked potency compared with $\mathrm{P} 3$ in vitro, and the antitumor efficacy of P3 and P27 against OSCC xenografts in athymic nude mice was investigated. In order to identify the antiproliferative mechanism of action, the effects of P3 and P27 on cell cycle distribution, mitochondrial transmembrane potential and apoptosis in vitro were investigated. To identify potential TRPV1-associated adverse effects, the effects of P3 and P27 on TRPV1 channel activation in vitro and nocifensive behaviors elicited in a rat model of orofacial pain were investigated.

\section{Materials and methods}

Plant material. Fresh Tasmannia lanceolata leaf material was provided by Essential Oils of Tasmania, Kingston, Tasmania, Australia (www.eotasmania.com.au) and dried at $35^{\circ} \mathrm{C}$ for $18 \mathrm{~h}$ before use. This leaf material originated from trees cultivated as commercial T. lanceolata crops grown in Tasmania, Australia. Polygodial was isolated from T. lanceolata following a procedure described previously (10).

Synthesis. Compound P3 was prepared as described previously (2). Compound P27 was prepared using the following procedure (Fig. 1A): To a solution of tetraethyl methylenediphosphonate $(36.9 \mathrm{mg}, 0.128 \mathrm{mmol})$ in tetrahydrofuran (THF; $2 \mathrm{ml}), 1.6 \mathrm{M}$ n-butyllithium $(80 \mu 1,0.128 \mathrm{mmol})$ was added at $-78^{\circ} \mathrm{C}$, which was then stirred for $20 \mathrm{~min}$. A solution of polygodial $(10 \mathrm{mg}, 0.0427 \mathrm{mmol})$ in THF (1 ml) (10) was then added dropwise at $-78^{\circ} \mathrm{C}$. The resultant mixture was allowed to warm up to $0^{\circ} \mathrm{C}$ and stirred for $2 \mathrm{~h}$. Following completion, the reaction was quenched with saturated $\mathrm{NH}_{4} \mathrm{Cl}$ and then extracted with ethyl acetate. The organic layer was washed with water and dried over anhydrous $\mathrm{Na}_{2} \mathrm{SO}_{4}$, and then concentrated to give a crude residue. The crude product was purified by preparative thin-layer chromatography using a $60 \%$ ethyl acetate/hexanes solvent system and P27 was obtained with a 95\% yield as a mixture of $\mathrm{C}_{9}$-epimers $(14.9 \mathrm{mg}$ ). The previously synthesized compound P3 and the novel compound P27 were each $>95 \%$ pure on the basis of the NMR spectra.

Characterization data. ${ }^{1} \mathrm{H}$ NMR $\left(400 \mathrm{MHz}, \mathrm{C}^{2} \mathrm{HCl}_{3}\right) \delta 9.57$ $(1 \mathrm{H}, \mathrm{d}, J 5.0 \mathrm{~Hz}), 9.47(0.5 \mathrm{H}, \mathrm{d}, J 4.8 \mathrm{~Hz}), 7.23-7.00(1.5 \mathrm{H}$, m), 6.51-6.41 (1.5 H, m), $5.57(1 \mathrm{H}, \mathrm{t}, J 17.4 \mathrm{~Hz}), 5.26(0.5 \mathrm{H}, \mathrm{t}$, $J$ 17.1 Hz), 4.12-3.94 (6 H, m), 2.88-2.71 (1.5 H, m), $2.49(1 \mathrm{H}$, $\mathrm{dt}, J 20.2,5.0 \mathrm{~Hz}), 2.31(0.5 \mathrm{H}, \mathrm{dt}, J 19.8,4.8 \mathrm{~Hz}), 2.27-2.13$ (1.0 H, m), 1.89-1.80 (0.5 H, m), 1.78-1.64 (3 H, m), 1.63-1.55 (1.5 H, m), 1.54-1.45 (3 H, m), 1.35-1.25 (9 H, m), 1.23-1.14 (3 H, m), 1.02-0.87 (13.5 H, m); ${ }^{13} \mathrm{C} \mathrm{NMR} \mathrm{(100} \mathrm{MHz,} \mathrm{C}^{2} \mathrm{HCl}_{3}$ ) ઈ 205.4, 201.7, 150.6, 150.5, 150.0, 149.9, 141.2, 141.2, 141.0, $140.9,130.9,130.7,129.3,129.0,113.13,113.1,111.3,111.2$, 62.6, 62.6, 62.2, 62.1, 61.5 (m) 60.4, 48.6, 44.6, 42.1, 41.8, 40.2,
37.7, 37.4, 36.6, 33.2, 33.1, 32.7, 25.4, 25.46, 24.7, 24.7, 22.2, 21.9, 21.2, 21.0, 18.4, 18.0, 16.4 (m), 15.5, 14.2; high-resolution mass spectrometry $m / z$ (electrospray ionization) for $\mathrm{C}_{20} \mathrm{H}_{34} \mathrm{O}_{4} \mathrm{P}$ $\left(M+\mathrm{H}^{+}\right)$calculated, 369.2195; found, 369.2193.

Cell lines. All cell lines, namely Cal27 (OSCC), PC3 (prostate cancer), HeLa (cervical cancer) and H460 (non-small cell lung cancer; NSCLC), were obtained from the American Type Culture Collection (Manassas, VA, USA). HSC3 cells were provided by Dr. Brian Schmidt (New York University College of Dentistry, New York, NY, USA). Genetica DNA Laboratories (Cincinnati, OH, USA) authenticated all cell lines prior to use in the present study. Cells were maintained in Dulbecco's modified Eagle's medium (DMEM; Gibco; Thermo Fisher Scientific, Inc., Waltham, MA, USA) supplemented with $10 \%$ fetal bovine serum and $1 \%$ penicillin/streptomycin, and maintained at $37^{\circ} \mathrm{C}$ under $5 \% \mathrm{CO}_{2}$. In order to determine if P3 and P27 interact with TRPV1 channels, CHO cells overexpressing TRPV1 (CHO-TRPV1) were used for calcium imaging studies. CHO-TRPV1 cells were kindly provided by Dr. Ardem Patapoutian at the Scripps Research Institute, and cultured as previously described at $37^{\circ} \mathrm{C}$ under $5 \% \mathrm{CO}_{2}(11)$.

MTS cell viability assays. Cell viability was assessed using a Cell Titer $96^{\circledR}$ Aqueous Non-Radioactive Cell Proliferation assay (Promega Corporation, Madison, WI, USA), according to the manufacturer's protocol. For concentration curves, cells were plated and treated for $48 \mathrm{~h}$ with increasing concentrations of polygodial, P3 or P27 diluted in DMEM at the indicated concentrations, as described previously (12). Pretreatments were performed using capsazepine $(\mathrm{CPZ} ; 1 \mu \mathrm{M})$ or $\mathrm{N}$-acetylcysteine (NAC; $10 \mathrm{mM}$ ) for $30 \mathrm{~min}$, followed by co-treatment with $25 \mu \mathrm{M}$ polygodial, P3 or P27 for $24 \mathrm{~h}$. Final ethanol concentrations were maintained at $0.1 \%$. Absorbance values of the test groups were compared with those of the vehicle-treated controls ( $\mathrm{n}=4$ per group).

Antitumor evaluations. All mouse studies were approved by the University of Texas Health Science Center at San Antonio (UTHSCSA) Institutional Animal Care and Use Committee (IACUC). All animal studies followed the international guidelines on animal welfare and were in accordance with the National Institutes of Health (NIH) guide for the care and use of laboratory animals. In addition, all animal studies complied with the Animal Research: Reporting of In Vivo Experiments (ARRIVE) guidelines and the 2013 American Veterinary Medical Association (AVMA) euthanasia guidelines. A total of 20 6-week-old female athymic nude mice, each weighing approximately $16 \mathrm{~g}$ (Envigo Laboratories, Indianapolis, IN, USA), were used in a laminar airflow cabinet under pathogen-free conditions. Mice were provided with a 12-h light/12-h dark schedule at a controlled temperature and humidity, with food and water available ad libitum. Mice were acclimated for 1 week before study initiation.

Mice were subcutaneously injected in the right flank with $3 \times 10^{6}$ Cal27 cells in $0.1 \mathrm{ml}$ sterile PBS. At 2-4 weeks postinoculation, tumors grew to an average volume of $100 \mathrm{~mm}^{3}$ and mice were stratified into four experimental groups $(n=5$ per group), receiving one of the following treatments via intratumor injection: Vehicle control (100 $\mu 10.25 \%$ ethanol 
diluted in sterile saline), $120 \mu \mathrm{g}$ polygodial, $\mathrm{P} 3$ or P27 diluted in $100 \mu \mathrm{l}$ sterile saline (final concentration of $0.25 \%$ ethanol). Treatments were repeated every other day for 3 weeks. Mice were monitored daily for tumor growth (using digital calipers), cachexia and weight loss. Tumor volumes were calculated using the elliptical formula: $1 / 2$ (length $\mathrm{x}$ width ${ }^{2}$ ) and the maximum allowable tumor volume was $1.5 \mathrm{~cm}^{3}$; however the largest tumor volume in this study was $510 \mathrm{~mm}^{3}$ (13). At the conclusion of the experiment, tumors were formalin-fixed and processed for histological analysis. Hematoxylin and eosin (H\&E) staining, terminal deoxynucleotidyl transferase dUTP nick end labeling (TUNEL) and Ki-67 staining (Ventana Medical Systems, Inc., Tucson, AZ, USA) were performed to detect apoptotic figures and proliferating cells respectively; these analyses were conducted by the UTHSCSA South Texas Reference Laboratory Histopathological Core Facility using standard methods.

Calcium imaging. Calcium imaging was performed using a FLIPR Calcium 6 Evaluation Kit (Molecular Devices, LLC, Sunnyvale, CA, USA), according to the manufacturer's protocol. CHO-TRPV1 cells $\left(9 \times 10^{3}\right)$ were seeded into a 384-well plate, loaded with Calcium 6 dye for $2 \mathrm{~h}$ and assessed using a PHERAstar FS multimode plate reader (BMG Labtech, Cary, NC, USA). The effects of $5 \mu \mathrm{M}$ P3 and P27 ( $\mathrm{n}=3$ per group) on calcium influx were determined according to alterations in fluorescent intensity $(535 \mathrm{~nm})$ and compared with that of $100 \mathrm{nM}$ capsaicin (positive control).

Eye-wipe testing. All procedures for the rat studies were approved by the UTHSCSA IACUC and followed the NIH Guidelines for the Care and Use of Laboratory Animals. In addition, all rat studies complied with the ARRIVE guidelines and the 2013 AVMA euthanasia guidelines. Six-week-old male Sprague-Dawley rats, weighing approximately $300 \mathrm{~g}$ (Envigo Laboratories), were provided with a 12-h light/12-h dark schedule at a controlled temperature and humidity, with food and water available ad libitum. Rats were acclimated for two weeks prior to study initiation. Rats were placed in a temperature-controlled $\left(22-25^{\circ} \mathrm{C}\right)$ behavioral laboratory in individual mirrored testing boxes $(30 \times 30 \times 30 \mathrm{~cm})$ in which they were allowed to acclimate for at least $1 \mathrm{~h}$. One drop (40 $\mu \mathrm{l}$ ) of a solution of $0.01 \%$ (w/v) capsaicin, P3, or P27 in sterile saline, was dropped onto one eye of each freely moving animal ( $n=6$ per group), as described previously $(14,15)$. The time spent grooming or closing the affected eye was recorded for a total of $2 \mathrm{~min}$, with the observers blinded to the treatment allocation groups.

Cell cycle distribution. Cal27 cells were cultured to $50 \%$ confluency and treated with $25 \mu \mathrm{M}$ polygodial, $\mathrm{P} 3$ or $\mathrm{P} 27$ for $24 \mathrm{~h}$. Cells were harvested and fixed in $70 \%$ ethanol followed by treatment with RNase A and staining with propidium iodide. Fluorescence-activated cell sorting (FACS) analysis of DNA profiles in terms of the proportion of cells in the $G_{1}, S$ and $\mathrm{G}_{2} / \mathrm{M}$ phases of the cell cycle was performed.

$J C-1$ assay. Cal27 cells were plated at a density of $5 \times 10^{5}$ in 60-mm dishes and grown overnight. Medium was replaced the following day with phenol red-free DMEM supplemented with $20 \mathrm{mM}$ 4-(2-hydroxyethyl)-1-piperazine-ethanesulfonic acid buffer to maintain a proper $\mathrm{pH}$ of 7.4. Cells were then treated for $2 \mathrm{~h}$ with polygodial, P3, P27, or vehicle control ( $n=3$ per group) at the indicated concentrations. As a positive control, cells were also treated with $15 \mu \mathrm{M}$ carbonyl cyanide m-chlorophenyl hydrazide (CCCP). Cells were then stained with $1 \mu \mathrm{M} \mathrm{JC}-1$ dye (Thermo Fisher Scientific, Inc.) for $15 \mathrm{~min}$ at $37^{\circ} \mathrm{C}$, washed, and assessed via FACS analysis.

Western blot analysis. Cal27 cells were treated for $48 \mathrm{~h}$ with the vehicle control, or with 10,25 and $50 \mu \mathrm{M}$ polygodial, P3 or P27 (final ethanol concentration of $0.1 \%$ ), respectively, and then harvested and lysed in Laemmli lysis buffer. The cell lysate concentration was determined at $570 \mathrm{~nm}$ reading using a bicinchoninic acid protein assay (Pierce; Thermo Fisher Scientific, Inc.), according to the manufacturer's protocol. Cell lysates containing equal concentrations of protein $(40 \mu \mathrm{g})$ were separated by SDS-PAGE (10\% gel), before being transferred onto a PVDF membrane and blocked in 5\% milk solution in PBS with $0.05 \%$ Tween-20. The membrane was incubated overnight at $4{ }^{\circ} \mathrm{C}$ with anti-cleaved poly(ADP-ribose) polymerase (c-PARP) rabbit polyclonal antibody (cat. no. 5625S; dilution, $1: 1,250)$, anti- $\alpha / \beta$-tubulin rabbit monoclonal antibody (cat. no. CS2148; dilution, 1:1,000) and anti-GAPDH mouse monoclonal antibody (cat. no. 97166S; dilution, 1:1,000; all from Cell Signaling Technology, Inc., Danvers, MA, USA) in a total of $6 \mathrm{ml}$ diluent (1\% milk in PBS containing $0.1 \%$ Tween-20), as previously described (12). The membrane was washed five times with PBS containing Tween-20 and incubated with Enhanced Chemiluminescence Plus detection solution (GE Healthcare, Chicago, IL, USA) for $1 \mathrm{~min}$. Signals were detected by exposure to radiographic film for $30 \mathrm{sec}$.

Statistical analysis. Statistical analyses were performed using GraphPad Prism (version 4; GraphPad Software, Inc., La Jolla, CA, USA). Results from cell viability, calcium influx, nocifensive behavior and mitochondrial transmembrane potential assays, in addition to Ki-67 staining and TUNEL assays $(n=3$ per group; 3 fields per section), were analyzed using one-way analysis of variance (ANOVA) and Bonferroni's post-hoc test. Statistical analyses of tumor growth were conducted using two-way ANOVA and repeated measures with Bonferroni's post-hoc test. Cell cycle distribution was analyzed using Student's t-test. All data are expressed as the mean \pm standard deviation, except for the cell cycle distribution results, which are expressed as the mean \pm standard error of the mean. $\mathrm{P}<0.05$ was considered to indicate a statistically significant difference.

\section{Results}

P27, a novel polygodial analog exhibits potent antiproliferative effects in vitro. P27 was synthesized and purified to $95 \%$, as outlined in Fig. 1A, prior to analysis for its effects against multiple cancer types using cell viability assays: Cal27 (OSCC), HSC3 (OSCC), PC3 (prostate cancer), HeLa (cervical cancer) and H460 (NSCLC). It was identified previously that the antiproliferative effects of polygodial and P3 are not cancer-type-specific (2). Likewise, P27 was efficacious against multiple cancer types resulting in a concentration-dependent decrease in cell viability following $48 \mathrm{~h}$ of treatment, with the 
A

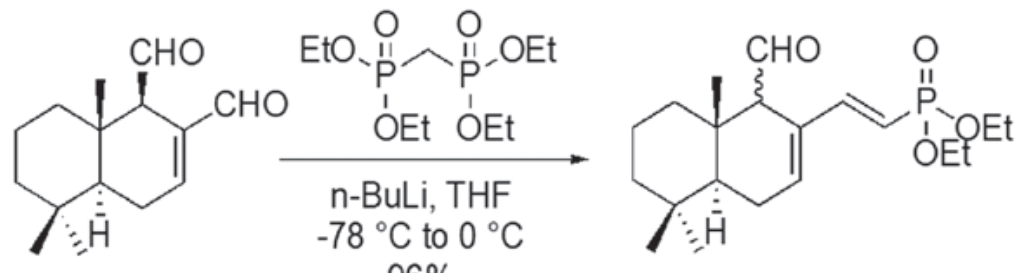

$96 \%$

DR-P27

B

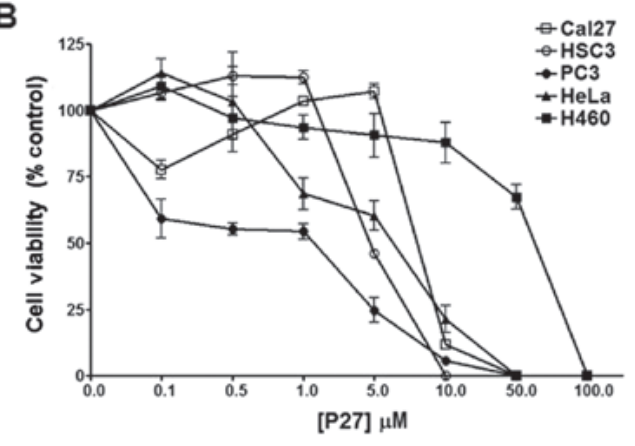

D

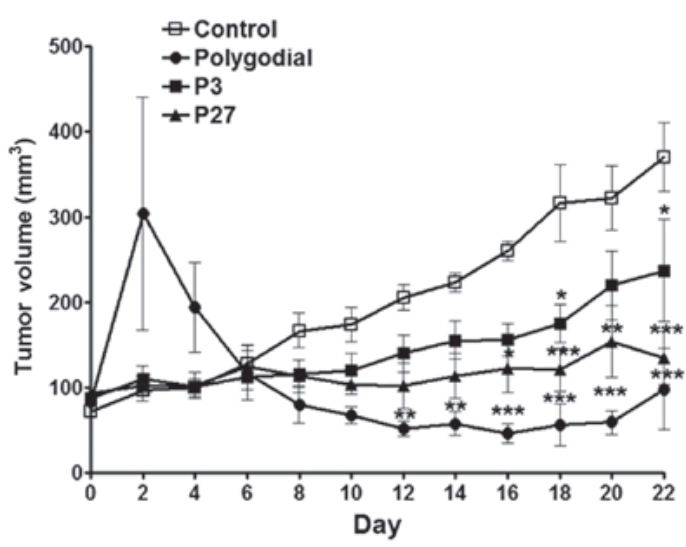

C

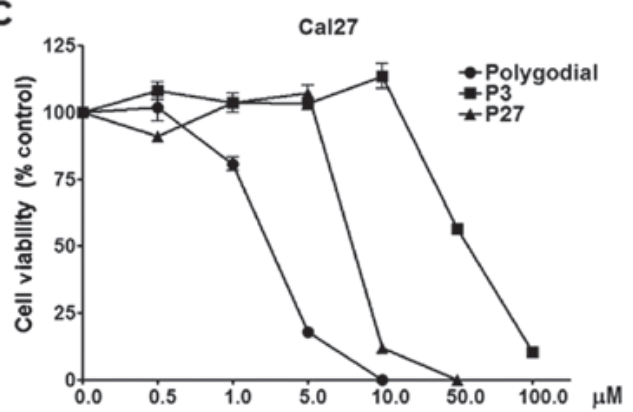

E

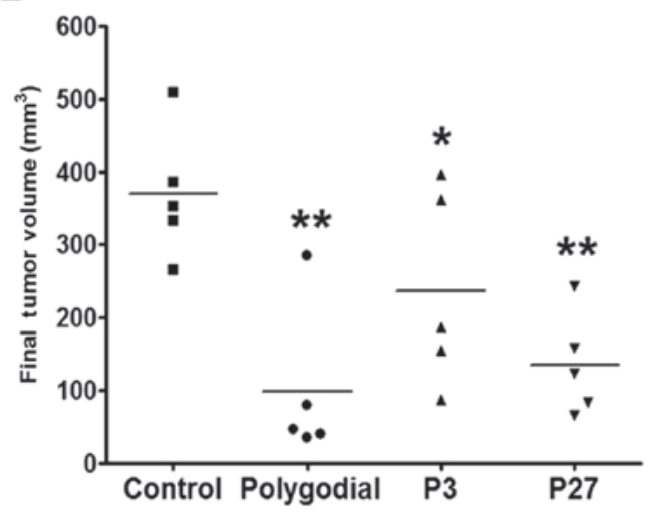

Figure 1. Effects of polygodial analogs on cancer cell proliferation in vitro and tumor growth in vivo. (A) Schematic diagram of P27 synthesis. (B) MTS viability assay in cancer cell lines treated with P27 for $48 \mathrm{~h}$ ( $\mathrm{n}=4$ per group). (C) MTS viability assays of Cal27 cells treated with polygodial, P3 or P27 for $48 \mathrm{~h}$. (D) Tumor volumes (mean \pm standard deviation) of Cal27-derived xenografts treated with $120 \mu \mathrm{g}$ polygodial, P3 or P27 every other day for 22 days. Significant decreases in tumor volumes were observable by day 12 for polygodial, day 16 for $\mathrm{P} 27$ and day 18 for $\mathrm{P} 3$ ( $\mathrm{n}=5$ per group; ${ }^{*} \mathrm{P}<0.05$, ** $\mathrm{P}<0.01$ and ${ }^{* * *} \mathrm{P}<0.001$ vs. vehicle control). (E) Scatter plot of Cal27 tumor volumes. The mean volume for vehicle control-treated tumors was $370 \mathrm{~mm}^{3}$, whereas the mean tumor volumes for polygodial, P3 and P27 treated tumors were 98,237 and $135 \mathrm{~mm}^{3}$, respectively ( $\mathrm{n}=5$ per group; ${ }^{*} \mathrm{P}<0.05$ and $\left.{ }^{* *} \mathrm{P}<0.01\right)$. Et, ethyl; $\mathrm{n}-\mathrm{BuLi}$, n-butyllithium; THF, tetrahydrofuran.

concentration of drug causing $50 \%$ decrease in proliferation of cancer cells $\left(\mathrm{GI}_{50}\right)$ ranging between 2 and $8 \mu \mathrm{M}$ in all cell lines except for $\mathrm{H} 460$ cells, which had a $\mathrm{GI}_{50}$ of $70 \mu \mathrm{M}$ (Fig. 1B).

P27 and P3 exhibit significant antiproliferative effects in Cal27 OSCC cells with no observable adverse side effects in tumor-bearing mice. To determine whether the antiproliferative effects observed in vitro translated in vivo, polygodial, $\mathrm{P} 3$ and P27 were investigated in Cal27 cells in culture and in Cal27-derived tumors xenografted in athymic nude mice. A marked decrease in Cal27 cell viability was noted following $48 \mathrm{~h}$ of treatment with polygodial, P3 and P27, with $\mathrm{GI}_{50}$ values of 4, 75 and $10 \mu \mathrm{M}$, respectively (Fig. 1C). Similarly, Cal27-derived tumors exhibited significant decreases in growth when treated with polygodial, P3 and P27 every other day (Fig. 1D). Notably, polygodial treatment initially induced a significant increase in tumor volume on day 2 that subsided by day 6. This increase was due to an inflammatory response at the injection site; however, no changes in activity or body weight were observed. One mouse exhibited some tissue damage (hemorrhage) following initial treatment with polygodial, which was resolved by day 6 . By day 12 , polygodial treatment had induced a significant decrease in tumor growth $(\mathrm{P}<0.01)$, compared with the vehicle control, an effect that persisted throughout the 22-day study period; final average tumor volumes were $98 \mathrm{~mm}^{3}$ and $370 \mathrm{~mm}^{3}$, respectively (Fig. 1E).

P3 and P27 treatments each led to significant decreases in tumor growth, with final average tumor volumes of $237 \mathrm{~mm}^{3}$ and $135 \mathrm{~mm}^{3}$, respectively (Fig. 1D and E). Similar to the in vitro results, P27 was more potent compared with P3 and exhibited significant decreases in tumor volumes by day 18 $(\mathrm{P}<0.001)$. There was no statistically significant difference between P27 and polygodial antitumor effects; the treatments yielded significantly decreased tumor volumes compared with 
A

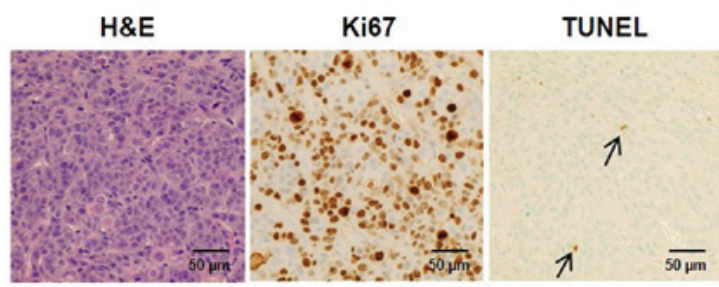

Poly

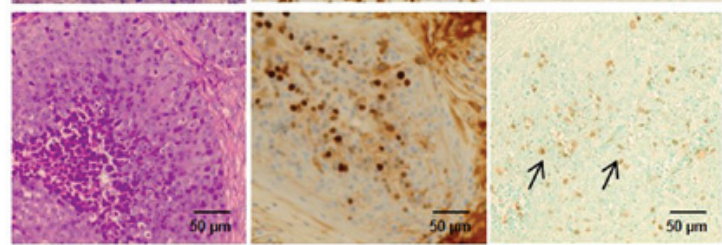

P3
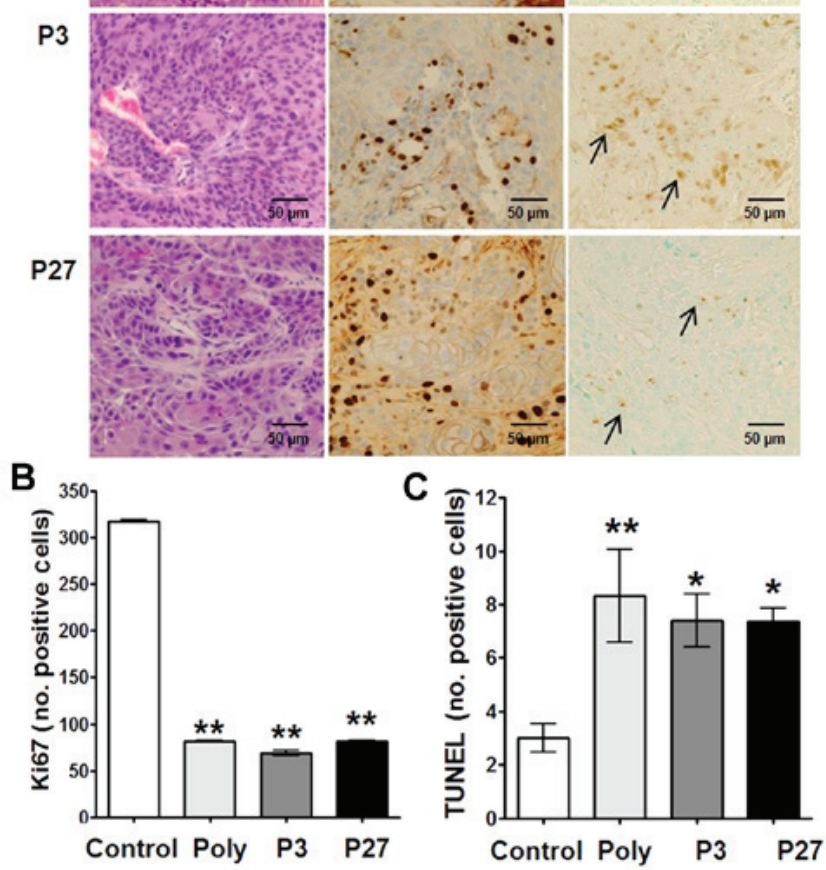

Figure 2. Histopathological analyses of Cal27-derived tumors treated with polygodial, P3 or P27. (A) Representative photomicrographs of Cal-27 derived tumors (magnification, $\mathrm{x} 40$ ) stained with $\mathrm{H} \& \mathrm{E}$ or $\mathrm{Ki}-67$, and from TUNEL assays; apoptotic cells are indicated with arrows. (B) Quantification of Ki-67-positive cells in treated tumors (mean $\pm \mathrm{SD}$; $=3$ per group; 3 fields/specimen; ${ }^{* *} \mathrm{P}<0.01$ vs. control). (C) Quantification of apoptotic cells in treated tumors (mean $\pm \mathrm{SD} ; \mathrm{n}=3$ per group; 3 fields/specimen; ${ }^{*} \mathrm{P}<0.05$ and ${ }^{* *} \mathrm{P}<0.01$ vs. control). H\&E, hematoxylin and eosin; TUNEL, terminal deoxynucleotidyltransferase dUTP nick end labeling; SD, standard deviation; Veh, vehicle; Poly, polygodial.

the vehicle control, and were equally efficacious. However, P27 did not elicit the inflammatory response that was consistently observed with polygodial, nor did it cause any observable tissue damage. Although P3 was comparably the least effective, it did significantly decrease tumor growth by day 22 when compared with the vehicle-treated controls $(\mathrm{P}<0.05)$; $\mathrm{P} 3$ treatment also had no detectable adverse effects.

Polygodial, P3, and P27 decrease the number of proliferating tumor cells and induce apoptosis in vivo. Histopathological analysis of H\&E-stained Cal27-derived tumors from mice treated with polygodial, P3 or P27 revealed large necrotic cores with viable cells along the tumor margins (Fig. 2A). Ki-67 staining and TUNEL assays were performed and quantified within the growing tumor front to assess changes
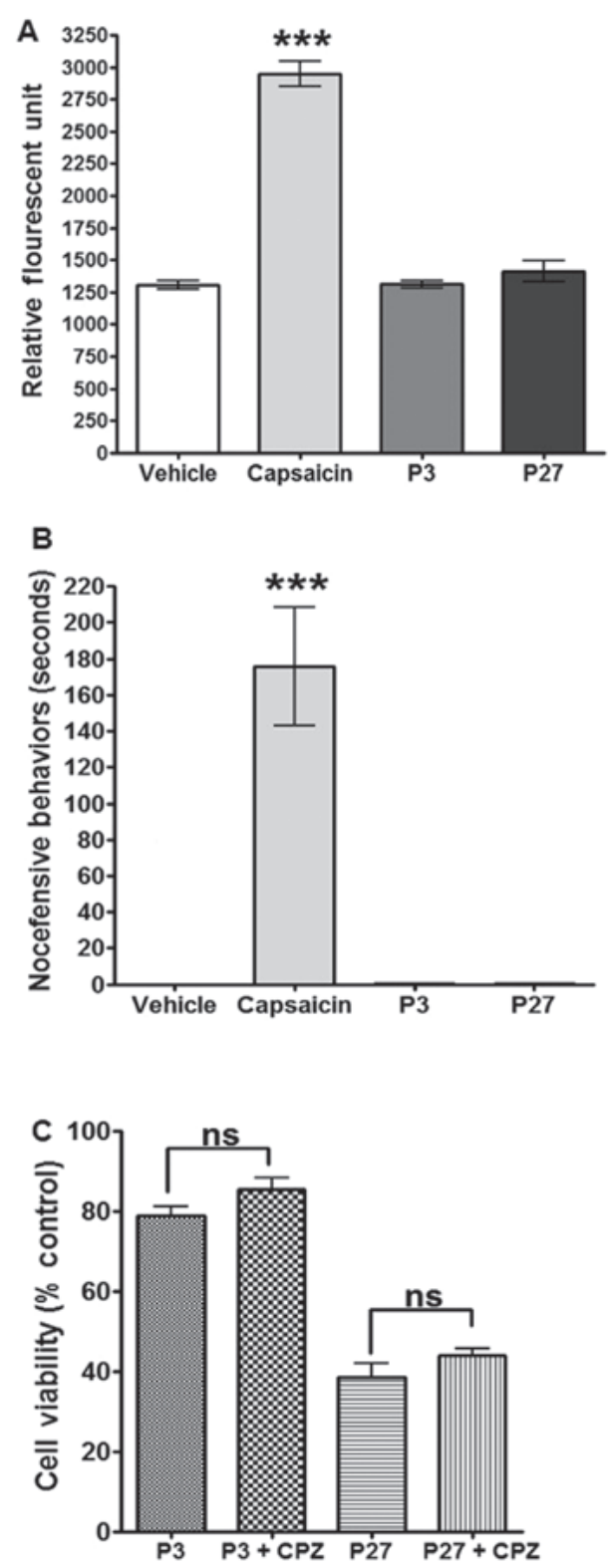

Figure 3. Evaluation of $\mathrm{P} 3$ and $\mathrm{P} 27$ activation of TRPV1 in vitro and in vivo. (A) Calcium imaging of CHO-TRPV1 cells treated with P3 $(5 \mu \mathrm{M}), \mathrm{P} 27(5 \mu \mathrm{M})$ or capsaicin positive control $(100 \mathrm{nM})$. Calcium influx as determined by mean fluorescence intensity $( \pm \mathrm{SD})$ is presented. (B) Eye-wipe testing in response to $0.01 \%(w / v) ~ P 3$, P27 or capsaicin ( $n=6$ per group). Nocifensive behavior measured in sec (mean \pm SD) is presented. (C) MTS viability assay of Cal27 cells Pretreated with CPZ $(1 \mu \mathrm{M})$, followed by P3 $(25 \mu \mathrm{M})$ or P27 $(25 \mu \mathrm{M})$ for $24 \mathrm{~h}$ ( $\mathrm{n}=4$ per group); ${ }^{* * *} \mathrm{P}<0.001$. TRPV1, transient receptor potential channel vanilloid subtype 1 ; CPZ, capsazepine; $\mathrm{SD}$, standard deviation.

in cell proliferation and apoptosis, respectively (Fig. 2A). All compounds significantly decreased the number of proliferating cells $(\mathrm{P}<0.01 ;$ Fig. $2 \mathrm{~B})$. In addition, a significant increase in the number of apoptotic cells, compared with the vehicle control, was observed with polygodial, P3 and P27 (Fig. 2C). Whereas polygodial yielded the highest number of apoptotic cells, there was no significant difference between polygodial, P3 and P27.

P3 and P27 fail to activate TRPVI in vitro and to induce nocifensive behavior in a rat model of orofacial pain. Our previous study indicated that P3 does not interact with TRPV1, 

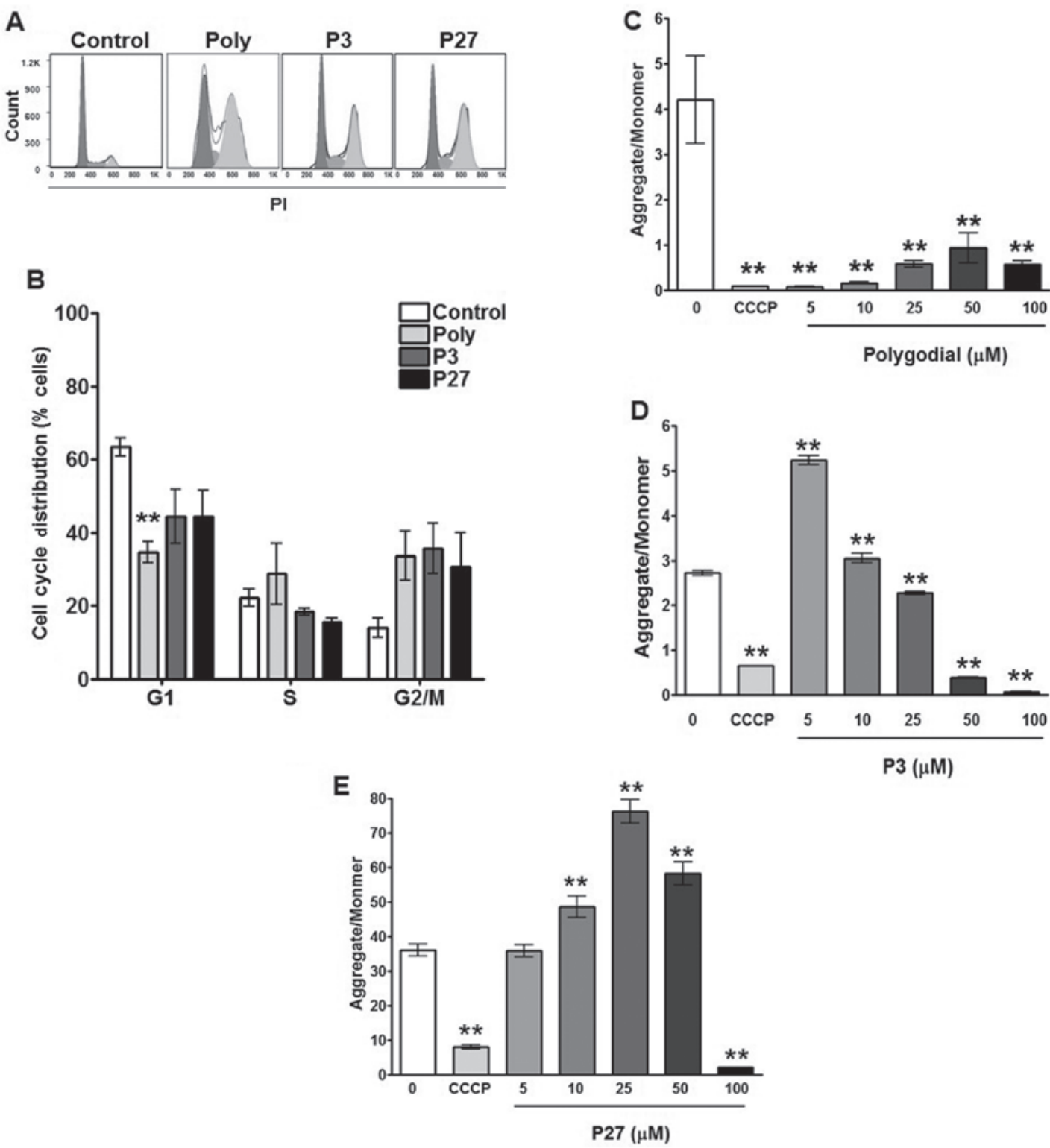

P3 $(\mu \mathrm{M})$ ment with $25 \mu \mathrm{M}$ polygodial, P3 or P27 for $24 \mathrm{~h}$. (B) Quantification of cell cycle distribution (mean \pm standard error of the mean). Mitochondrial membrane potential analysis of Cal27 cells treated for $2 \mathrm{~h}$ with (C) polygodial, (D) P3 or (E) P27 at the indicated concentrations (5, 10, 25, 50 and 100 $\mu \mathrm{M}$ ). As a positive control cells were also treated with $15 \mu \mathrm{M}$ CCCP. Ratios of JC-1 aggregates to monomers were quantified (mean \pm standard deviation); $\mathrm{n}=3$ per group; * $\mathrm{P}<0.01$ vs. no treatment control. Poly, polygodial; CCCP, carbonyl cyanide m-chlorophenyl hydrazide.

whereas polygodial is a known TRPV1 agonist (2). Therefore, the potential TRPV1 interactions with P27 were compared with those with P3. Using CHO cells that overexpress TRPV1, calcium imaging analysis was performed, which confirmed that P3 does not induce calcium influx (Fig. 3A). Similarly, P27 failed to induce calcium influx (Fig. 3A). We hypothesized that these compounds may be antagonizing TRPV1, and, thus, Pretreated CHO-TRPV1 cells with either P3 or P27, followed by the standard TRPV1 agonist capsaicin; however, no TRPV1 antagonism was identified using P3 or P27 (data not shown). These results were recapitulated in vivo using a rat model of orofacial pain. Rats were treated with the vehicle control, P3, P27 or capsaicin, and nocifensive behavior was determined for 5 min. Capsaicin induced nocifensive eye-wiping and blinking for $160 \pm 40 \mathrm{sec}$. As with the vehicle control, P3 and P27 failed to induce nocifensive behavior (Fig. 3B).
Finally, to confirm that TRPV1 activation does not regulate the antiproliferative effects of P3 and P27, Cal27 cells were Pretreated with CPZ $(1 \mu \mathrm{M})$, followed by treatment with $\mathrm{P} 3$ or P27, and the effects on cell proliferation were analyzed. CPZ failed to reverse the antiproliferative effects of all treatments, and no additive effects were noted (Fig. 3C).

Polygodial, P3 and P27 induce a $G_{2} / M$ phase block and mitochondrial dysfunction in OSCC cells. In order to assess the TRPV1-independent mechanism(s)-of-action, cell cycle distribution and mitochondrial function assays were performed. Cell cycle distribution analysis in Cal27 cells revealed that polygodial, P3 and P27 cause a $\mathrm{G}_{2} / \mathrm{M}$ phase block (Fig. 4A). Quantification of the cell cycle distribution revealed a significant decrease in the number of polygodial-treated cells in the $G_{1}$ phase, with corresponding increases in the $S$ 


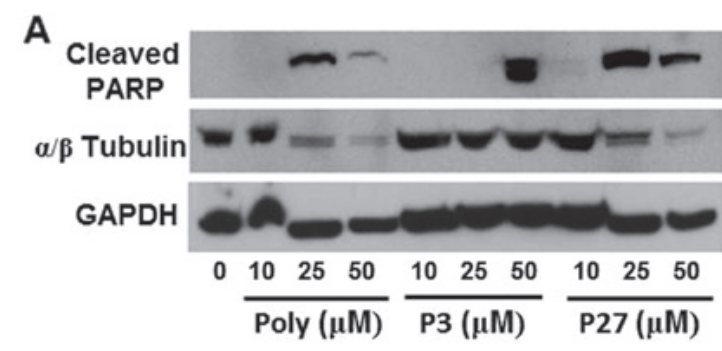

B
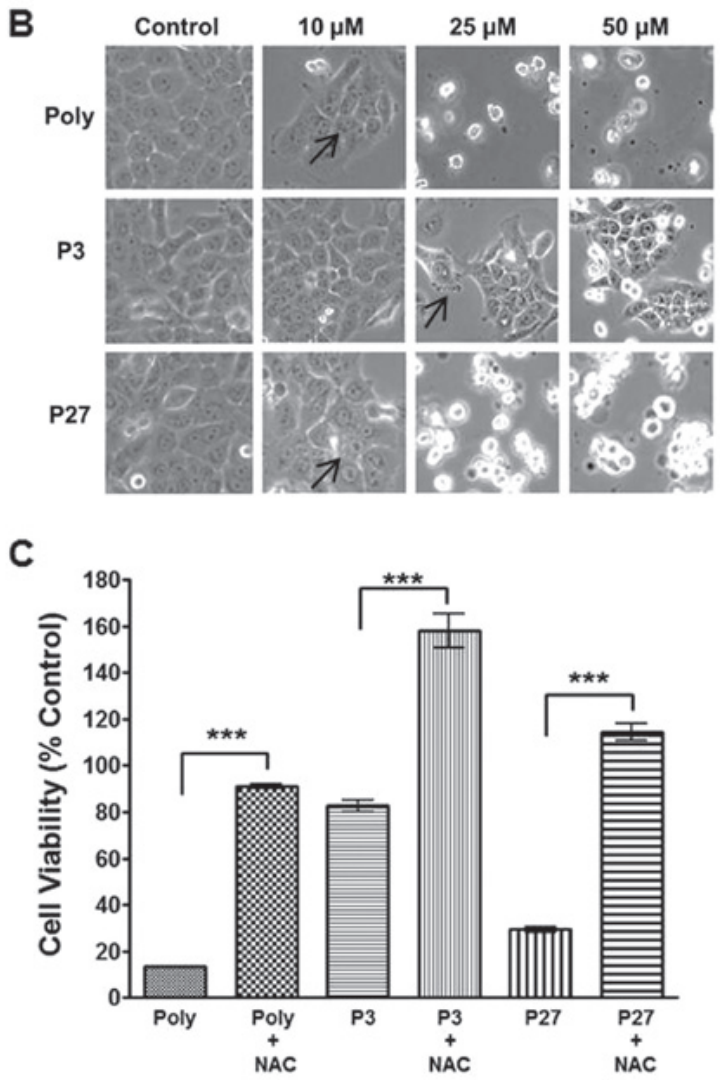

Figure 5. Effects of polygodial, P3 and P27 on apoptosis. (A) Western blot analysis of cleaved PARP in Cal27 cells treated with increasing concentrations of polygodial, P3 or P27 for $48 \mathrm{~h}$. (B) Photomicrographs (magnification, $\mathrm{x} 40$ ) of Cal27 cells treated with increasing concentrations of polygodial, P3 or P27 for 48 h. Membrane blebbing and apoptotic figures are indicated by black arrows. (C) MTS viability assays of Cal27 cells treated with $25 \mu \mathrm{M}$ polygodial, P3 or P27 with or without 10 mM NAC for $24 \mathrm{~h}(\mathrm{n}=4$ per group; $\left.{ }^{* * *} \mathrm{P}<0.001\right)$. PARP, poly(ADP-ribose) polymerase; NAC, N-acetylcysteine; Poly, polygodial.

and $\mathrm{G}_{2} / \mathrm{M}$ phases (Fig. 4B). P3 and P27 induced a decrease in the percentage of cells in the $\mathrm{G}_{1}$ phase and an increase in the percentage of cells in the $\mathrm{G}_{2} / \mathrm{M}$ phase, compared with the control; however, these differences were not statistically significant. Mitochondrial transmembrane depolarization was measured in response to increasing concentrations of polygodial, $\mathrm{P} 3$ and $\mathrm{P} 27$, using $\mathrm{JC}-1$ dye. The aggregate to monomer ratio, representative of mitochondrial transmembrane potential, was immediately diminished with even the lowest concentration of polygodial $(5 \mu \mathrm{M})$, indicating a significant decrease in transmembrane potential ( $\mathrm{P}<0.01$; Fig. 4C). Similarly, P3 and P27 treatments each elicited significant decreases in mitochondrial transmembrane potential, but at higher concentrations compared with polygodial (25 and $100 \mu \mathrm{M}$, respectively; Fig. 4D and E).
In order to determine whether the decrease in membrane potential paralleled the induction of apoptosis, western blot analysis of c-PARP in Cal27 cells was performed. Indeed, a concentration-dependent induction of c-PARP was present with all treatments (Fig. 5A). Similarly, photomicrographs of cells treated with polygodial, P3 and P27 identified membrane blebbing, apoptotic figures and apoptosis with increasing concentrations that corresponded to the induction of c-PARP (Fig. 5B; arrows). For example, polygodial and P27 induced c-PARP at concentrations of $25 \mu \mathrm{M}$, whereas P3 required a higher concentration $(50 \mu \mathrm{M})$ to induce this effect within the same timeframe (Fig. 5A). In addition, increased c-PARP levels were correlated with increased numbers of apoptotic cells as presented in the photomicrographs at $25 \mu \mathrm{M}$ for polygodial and P27, and at $50 \mu \mathrm{M}$ for P3.

Cell viability assays confirmed that the antioxidant NAC reversed the anti-proliferative effects of polygodial, P3 and P27 ( $\mathrm{P}<0.001$; Fig. 5C), implicating reactive oxygen species (ROS) as potential mediators of mitochondrial dysfunction and cell apoptosis. Additionally, a concentration-dependent decrease in tubulin (initially used as a loading control) was observed with polygodial and P27 treatments (Fig. 5A). Therefore, equal protein loading concentrations were established with GAPDH, which did not exhibit alterations in expression following these treatments, thus confirming the changes in tubulin levels in response to polygodial and $\mathrm{P} 27$ treatments.

\section{Discussion}

Numerous sesquiterpenes, including polygodial, are reported to have medicinal and antifeedant properties. Polygodial is an unsaturated sesquiterpene dialdehyde that is non-mutagenic with significant antimicrobial and cytotoxic activities against multiple cancer types $(9,16)$. Structure activity association studies of unsaturated sesquiterpene dialdehydes reveal that specific structural features of the dialdehyde functional group are essential for cytotoxicity (2,16-18). For example, it was identified that the removal of the aldehyde groups, through either their reduction to the diol or incorporation into the pyridazine ring, led to the loss of activity $(2,18)$. However, conversion of the $\alpha, \beta$-unsaturated aldehyde into an $\alpha, \beta-\gamma, \delta$-unsaturated ester resulted in a more potent analog, P3 (2). The present study involved the synthesis of the polygodial analog P27, which contains an $\alpha, \beta-\gamma, \delta$-unsaturated phosphonate group in place of the aldehyde (Fig. 1A) and maintains superior antiproliferative effects while avoiding the undesired TRPV1 agonist property associated with polygodial. On the basis of our chemical studies (2), we previously proposed that P3 exerts its biological activities through the formation of a covalent complex with a lysine group of its intracellular target (Fig. 6). Since similar chemistry should apply to P27 (Fig. 6), it could also be hypothesized that the reaction of $\mathrm{P} 27$ with a lysine residue to irreversibly form a stable pyrrole ring is responsible for its anticancer effects. This hypothesis requires further study.

As with polygodial, P27 cytotoxicity is not specific to any cancer type tested; rather, P27 is highly efficacious against multiple cancer types, indicating that it may have a universal anticancer mechanism of action. However, the present mechanistic study focused specifically on oral cancer. P27 significantly decreased the viability of two OSCC cell 
<smiles>[X]C=CC1=CC[C@H]2C(C)(C)CCC[C@]2(C)[C@H]1CO</smiles>

$\underset{\text { target-Lys- } \mathrm{NH}_{2}}{\longrightarrow}$

P3 $\mathrm{X}=\mathrm{CO}_{2} \mathrm{Me}$ or $\mathbf{P 2 7} \mathrm{X}=\mathrm{PO}(\mathrm{OEt})_{2}$

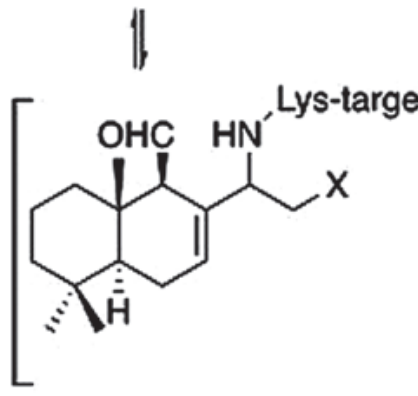

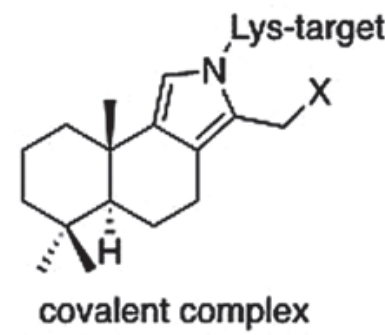
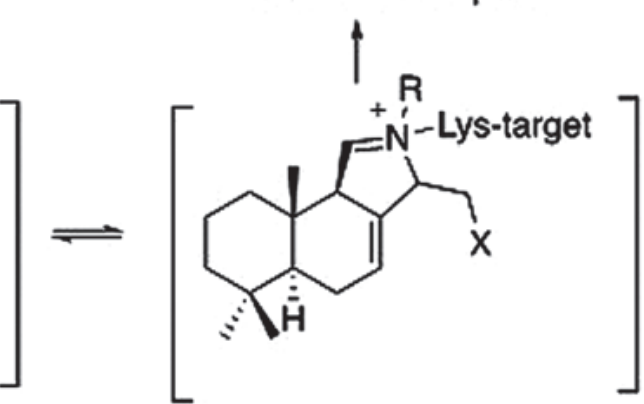

Figure 6. Schematic diagram for the hypothesized reaction of P27 with a lysine residue to irreversibly form a stable pyrrole ring as a mediator of P27 anticancer effects. Me, methyl; Et, ethyl.

lines, Cal27 and HSC3. Comparative analyses performed in Cal27 cells demonstrated that $\mathrm{P} 27$ was more potent compared with $\mathrm{P} 3$, and less potent compared with polygodial, in vitro. However, P27 was equipotent with polygodial against OSCC tumor growth in athymic nude mice revealing significant decreases in tumor volumes and proliferating cells, and significant increases in the number of apoptotic cells, compared with vehicle control-treated tumors. P3 was also identified to be efficacious against OSCC xenografts; however, similar to the cell based assays, P3 remained the least potent of the three compounds.

Polygodial consistently induced a marked yet transitory inflammatory response at the injection site in all mice, which was evident by day 2 and had subsided by day 6 . No respiratory distress was observed, and no changes in activity levels or body weight were noted with any of the treatments. However, one mouse did develop a hemorrhage at the injection site following administration of the first polygodial treatment, which healed and did not recur. Presumably, the inflammatory response and soft tissue damage was due to TRPV1 activation in adjacent non-cancerous tissues. P3 and P27 did not induce any observable adverse side effects in adjacent non-malignant tissues, respiration or neuromuscular function. Furthermore, behavioral studies using a rat orofacial pain model revealed that P3 and P27 do not elicit nocifensive behaviors, such as eye-wiping, blinking or closing of the eye, that are elicited with the TRPV1 agonist capsaicin. Polygodial was not analyzed in this assay because it is a known TRPV1 agonist and not a standard positive control for these behavioral studies. The aim is to develop novel therapeutic compounds based on the polygodial pharmacophore that do not elicit polygodial-associated adverse side effects. Indeed, calcium imaging confirmed that P3 and P27 do not activate or antagonize TRPV1 channels. However, future studies evaluating efficacy and toxicity via systemic administration are required to elucidate other potential adverse side effects.

In order to assess the anticancer mechanism(s)-of-action, cell cycle distribution analysis was performed. Notably, polygodial, P3 and P27 all caused a $\mathrm{G}_{2} / \mathrm{M}$ phase arrest. Although not the focus of the present study, it was also observed that polygodial and P27 significantly decreased tubulin protein levels, whereas P3 had no effect. The antifungal activity of polygodial is attributed, in part, to its binding to thiol groups found on fungal microtubules (19). Thus, future studies evaluating P27 effects on microtubule polymerization and cell cycle regulators in cancer are required in order to delineate this potential anticancer activity.

Polygodial also inhibits fungal mitochondrial ATP synthesis (7). Castelli et al (20) demonstrated that polygodial inhibits electron transport and ATPase activity in rat liver cells and bovine cardiac mitochondria in vitro. Therefore, the effects of polygodial, P3 and P27 on OSCC cell mitochondrial membrane potential were investigated as a measure of mitochondrial function. Mitochondrial transmembrane polarization is essential to drive ATP synthesis. Cancer cells are generally hyperpolarized, compared with non-cancerous cells, due to their high rate of respiration. Furthermore, high cellular respiration rates produce increased levels of ROS due to inefficiencies in the electron transport chain. We hypothesize that these compounds disrupt mitochondrial function, leading to high levels of ROS that surpass homeostatic thresholds and induce apoptosis. Indeed, the antioxidant NAC completely reversed the antiproliferative effects of polygodial, P3 and P27, implicating the induction of ROS as drivers of apoptosis.

Depolarization of the mitochondrial transmembrane potential is one of the key types of mitochondrial dysfunction that leads to apoptosis (21). It was identified that polygodial, P3 and P27 all induce depolarization of the mitochondrial transmembrane potential and cell apoptosis; however, the mechanisms may differ between polygodial and the analogs. Initially, P3 and P27 induced an increase in transmembrane potential followed by a concentration-dependent decrease in polarization. Calcium homeostasis is a regulator of cell death with the endoplasmic reticulum (ER) being the primary store of calcium in the cell, and the mitochondria the main effectors of calcium mobilization from the ER. These initial apparent 
increases in transmembrane potential are consistent with ER stress and the subsequent release of high calcium levels into the cytosol, thus interfering with the accuracy of dyes that provide indirect measures of mitochondrial $\mathrm{pH}$ (22). Furthermore, ROS are drivers of ER stress-associated calcium release, resulting in increased mitochondrial calcium levels and the production of additional ROS, thus creating a positive feedback loop that assures apoptosis (23-25). Therefore, P3-and P27-induced ROS may potentiate the apoptotic pathway via ER stress and subsequent mitochondrial transmembrane depolarization. Ultimately, all compounds tested induced mitochondrial transmembrane depolarization that corresponded with increased PARP cleavage and cell apoptosis; however, polygodial elicited the most notable depolarization of the mitochondrial membrane at even the lowest concentration tested $(5 \mu \mathrm{M})$. Polygodial has been identified to inhibit mitochondrial ATP synthase and ATPase; thus the marked depolarization of mitochondrial transmembrane potential may be due to polygodial's direct inhibition of ATP synthesis in addition to its promoting ROS production $(7,20)$. Conversely, higher concentrations of P3 and P27 were required to induce the same effect on mitochondrial transmembrane potential. Given that P27 is equipotent to polygodial in vivo, it may have a second mechanism of action that is responsible for its antitumor effects, which may include prompting ER stress. Studies are underway to evaluate the interactions OF P27 with the ER, electron transport complexes and microtubules in order to fully ascertain its anticancer mechanism(s)-of-action.

Taken together, we hypothesize that the shared anticancer effects of polygodial, P3 and P27 are due to the disruption of mitochondrial function, the generation of ROS and cell apoptosis. However, the novel compound P27 may have additional mechanism(s) that enhance its antitumor affects compared with P3; and with no observable adverse side effects that are present with polygodial. Additional studies evaluating higher doses and different routes of administration are underway in order to better target metastatic OSCC and to fully determine P27 efficacy as a single-agent therapy. Furthermore, P27 may prove to be useful as an adjuvant therapy to standard treatments, including chemotherapy and radiation therapy.

\section{Acknowledgements}

The authors would like to thank Karla M. Gorena for her assistance with the FACS analysis at the CTRC Flow Cytometry Core Facility.

\section{Funding}

The present study was supported by the University of Texas Health Science Center of San Antonio (UTHSCSA), Cancer Therapy and Research Center (CTRC). FACS analysis was performed at the CTRC Flow Cytometry Core Facility supported by a National Cancer Institute (NCI) Cancer Center Support Grant (grant no. P30 CA054174). Medicinal chemistry was supported by the NCI (grant no. CA186046-01A1). The study performed in the Center for Innovative Drug Discovery High Throughput Screening Facility was supported by the National Center for Advancing Translational Sciences, National Institutes of Health (grant no. UL1 TR001120).

\section{Availability of data and materials}

The datasets used and/or analyzed during the current study are available from the corresponding author on reasonable request.

\section{Authors' contributions}

All authors listed made substantial contributions to conception and design, acquisition of data, or analysis and interpretation of data. All authors were also involved in drafting the manuscript and have given final approval of the version to be published.

\section{Ethics approval and consent to participate}

All mouse and rat studies were approved by the University of Texas Health Science Center at San Antonio (UTHSCSA) Institutional Animal Care and Use Committee (IACUC). All animal studies followed the international guidelines on animal welfare and were in accordance with the National Institutes of Health (NIH) guide for the care and use of laboratory animals. In addition, all animal studies complied with the Animal Research: Reporting of In Vivo Experiments (ARRIVE) guidelines and the 2013 American Veterinary Medical Association (AVMA) euthanasia guidelines. All procedures for the rat studies were approved by the UTHSCSA IACUC and followed the NIH Guidelines for the Care and Use of Laboratory Animals. In addition, all rat studies complied with the ARRIVE guidelines and the 2013 AVMA euthanasia guidelines.

\section{Patient consent for publication}

Not applicable.

\section{Competing interests}

The authors declare that they have no competing interests.

\section{References}

1. Cancer Facts and Figures: American Cancer Society; 2016 \https://www.cancer.org/research/cancer-facts-statistics/all-cancerfacts-figures/cancer-facts-figures-2016.html Accessed on 11-13-17.

2. Dasari R, De Carvalho A, Medellin DC, Middleton KN, Hague F, Volmar MN, Frolova LV, Rossato MF, De La Chapa JJ, DybdalHargreaves NF, et al: Wittig derivatization of sesquiterpenoid polygodial leads to cytostatic agents with activity against drug resistant cancer cells and capable of pyrrolylation of primary amines. Eur J Med Chem 103: 226-237, 2015.

3. da Cunha FM, Fröde TS, Mendes GL, Malheiros A, Cechinel Filho V, Yunes RA and Calixto JB: Additional evidence for the anti-inflammatory and anti-allergic properties of the sesquiterpene polygodial. Life Sci 70: 159-169, 2001.

4. McCallion RF, Cole AL, Walker JR, Blunt JW and Munro MH: Antibiotic substances from New Zealand plants. II. Polygodial, an anti-Candida agent from Pseudowintera colorata. Planta Med 44: 134-138, 1982.

5. Barnes CS and Loder JW: Structure of polygodial-a new sesquiterpene dialdehyde from polygonum hydropiper L. Aust J Chem 15: 322-327, 1962.

6. André E, Campi B, Trevisani M, Ferreira J, Malheiros A, Yunes RA, Calixto JB and Geppetti P: Pharmacological characterisation of the plant sesquiterpenes polygodial and drimanial as vanilloid receptor agonists. Biochem Pharmacol 71: 1248-1254, 2006.

7. Kubo I, Fujita K and Lee SH: Antifungal mechanism of polygodial. J Agric Food Chem 49: 1607-1611, 2001. 
8. Leonard CM and Viljoen AM: Warburgia: A comprehensive review of the botany, traditional uses and phytochemistry. J Ethnopharmacol 165: 260-285, 2015.

9. Montenegro I, Tomasoni G, Bosio C, Quiñones N, Madrid A, Carrasco H, Olea A, Martinez R, Cuellar M and Villena J: Study on the cytotoxic activity of drimane sesquiterpenes and nordrimane compounds against cancer cell lines. Molecules 19: 18993-19006, 2014.

10. Just J, Jordan TB, Paull B, Bissember AC and Smith JA: Practical isolation of polygodial from Tasmannia lanceolata: A viable scaffold for synthesis. Org Biomol Chem 13: 11200-11207, 2015.

11. Rooney L, Vidal A, D'Souza AM, Devereux N, Masick B, Boissel V, West R, Head V, Stringer R, Lao J, et al: Discovery, optimization, and biological evaluation of 5-(2-(trifluoromethyl) phenyl)indazoles as a novel class of transient receptor potential A1 (TRPA1) antagonists. J Med Chem 57: 5129-5140, 2014.

12. Gonzales CB, De La Chapa JJ, Saikumar P, Singha PK, DybdalHargreaves NF, Chavez J, Horning AM, Parra J and Kirma NB: Co-targeting ALK and EGFR parallel signaling in oral squamous cell carcinoma. Oral Oncol 59: 12-19, 2016.

13. Jensen MM, Jørgensen JT, Binderup T and Kjaer A: Tumor volume in subcutaneous mouse xenografts measured by microCT is more accurate and reproducible than determined by $18 \mathrm{~F}-\mathrm{FDG}-$ microPET or external caliper. BMC Med Imaging 8: 16, 2008

14. Austah ON, Ruparel NB, Henry MA, Fajardo RJ, Schmitz JE and Diogenes A: Capsaicin-sensitive innervation modulates the development of apical periodontitis. J Endod 42: 1496-1502, 2016

15. Karai L, Brown DC, Mannes AJ, Connelly ST, Brown J, Gandal M, Wellisch OM, Neubert JK, Olah Z and Iadarola MJ: Deletion of vanilloid receptor 1-expressing primary afferent neurons for pain control. J Clin Invest 113: 1344-1352, 2004.

16. Anke $\mathrm{H}$ and Sterner O: Comparison of the antimicrobial and cytotoxic activities of twenty unsaturated sesquiterpene dialdehydes from plants and mushrooms. Planta Med 57: 344-346, 1991.
17. Forsby A, Walum E and Sterner O: The effect of six sesquiterpenoid unsaturated dialdehydes on cell membrane permeability in human neuroblastoma SH-SY5Y cells. Chem Biol Interact 84 : 85-95, 1992.

18. Dasari R, De Carvalho A, Medellin DC, Middleton KN Hague F, Volmar MN, Frolova LV, Rossato MF, De La Chapa JJ, Dybdal-Hargreaves NF, et al: Synthetic and biological studies of sesquiterpene polygodial: Activity of 9-epipolygodial against drug-resistant cancer cells. ChemMedChem 10: 2014-2026, 2015.

19. Kiso T, Fujita K, Ping X, Tanaka T and Taniguchi M: Screening for microtubule-disrupting antifungal agents by using a mitoticarrest mutant of Aspergillus nidulans and novel action of phenylalanine derivatives accompanying tubulin loss. Antimicrob Agents Chemother 48: 1739-1748, 2004.

20. Castelli MV, Lodeyro AF, Malheiros A, Zacchino SA and Roveri OA: Inhibition of the mitochondrial ATP synthesis by polygodial, a naturally occurring dialdehyde unsaturated sesquiterpene. Biochem Pharmacol 70: 82-89, 2005

21. Boland ML, Chourasia AH and Macleod KF: Mitochondrial dysfunction in cancer. Front Oncol 3: 292, 2013.

22. Perry SW, Norman JP, Barbieri J, Brown EB and Gelbard HA: Mitochondrial membrane potential probes and the proton gradient: A practical usage guide. Biotechniques 50: 98-115, 2011.

23. Haynes CM, Titus EA and Cooper AA: Degradation of misfolded proteins prevents ER-derived oxidative stress and cell death. Mol Cell 15: 767-776, 2004.

24. Malhotra JD, Miao H, Zhang K, Wolfson A, Pennathur S, Pipe SW and Kaufman RJ: Antioxidants reduce endoplasmic reticulum stress and improve protein secretion. Proc Natl Acad Sci USA 105: 18525-18530, 2008.

25. Zeeshan HM, Lee GH, Kim HR and Chae HJ: Endoplasmic Reticulum Stress and Associated ROS. Int J Mol Sci 17: 327, 2016. 